\title{
Utilization of Gravitation for Generation of Electricity as an Additional Source
}

\section{Soni NK*}

Bachelor Degree Program in Electrical and Electronics Engineering, Takshshila Institute of Engg. \& Tech, Jabalpur, India

\begin{abstract}
Under the discipline I have discussed about a concept to generate electricity using the gravitational force. A structure which is able to work itself for generating electricity, basic concept behind this is to utilize the structural and gravity both. A general structure and its arrangement for generation, various other aspects or application for the structure is being discussed.
\end{abstract}

Keywords: Electricity generation; Gravitational energy; Piezoelectric block; Balancing structure; Mechanical structure; Mechanical gear arrangement; Concentrating blocks

\section{Introduction}

Here are many Conventional and Non-Conventional source of Electricity and most of them are generally having an input for generation which cause in extra pollutants and burden one economy. Some fuel or source which are using for generation have their limit and limited efficiency, So as to have requirement of an arrangement that could help in generation of electricity, and this structure could help for the generation with no input [1]. One of the great aspects of this setup is can generate for $24 \times 7$ and doesn't depend upon weathered conditions. The design of this project comes to my mind when have seen an show piece structure, an generalized diagram is given below which exactly clear the picture of the system.

\section{Concept of the Gravitational Based System for Electricity Generation}

From the Figure 1 it's very clear that the concept or mechanism is the balance of the weight of the specimen and secondary is the flow of fluid, as the specimen weight concentrate downward (one side of the system). And another downward motion of the system is due to the design of the specimen so that when the system in pos 1 . The fluid flows downward to second position; that is there are two type of systems are working together which are balancing each other [2]. There are two alternative conditions that are illustrated as following Figures 2 and 3.

\section{Design of System for Generation}

There could be various design or method for generation i.e. We can use mechanical structure as well as Piezoelectric fundamental for this $[3,4]$. A block diagram for this using piezoelectric Structure is given Figure 4 . This mechanism is very well explained as, due to under gravity when the structure move downward right and left it

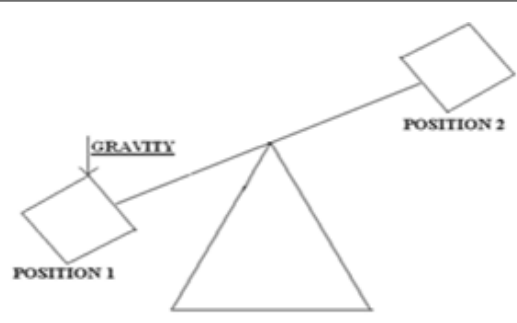

Figure 1: Block diagram of general concept. alternatively made pressure on the piezoelectric block which constitute current and which is then supplied to storage device or direct use.

An another mechanical structure for this is explained as following. The mechanical structure for gravitational based electricity generation concept is shown in Figure 5. Mechanical structure consist of two free wheels with center having mechanical gears arrangement which are connected to the shaft of the generator set. When the basic structure of the system made alternative downward motions having an Arrangement to rotate wheels so that cause in generation of electricity.

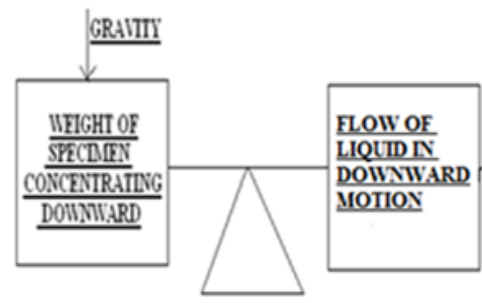

Figure 2: Block diagram of general concept.

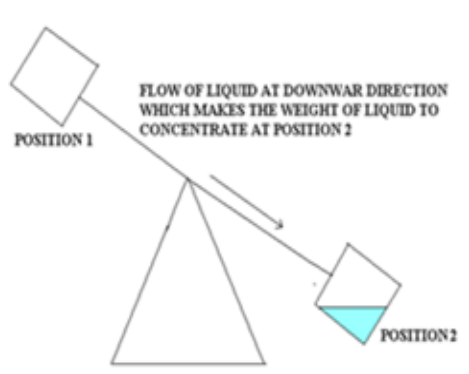

Figure 3: Block diagram of general concept.

*Corresponding author: Soni NK, Bachelor Degree Program in Electrical and Electronics Engineering, Takshshila Institute of Engineering and Technology, Jabalpur, India, Tel: 0761-2441352; E-mail: nayankumarsoni167@gmail.com

Received February 16, 2016; Accepted February 29, 2016; Published March 15 2016

Citation: Soni NK (2016) Utilization of Gravitation for Generation of Electricity as an Additional Source. J Electr Electron Syst 5: 177. doi:10.4172/2332-0796.1000177

Copyright: (c) 2016 Soni NK. This is an open-access article distributed under the terms of the Creative Commons Attribution License, which permits unrestricted use, distribution, and reproduction in any medium, provided the original author and source are credited. 


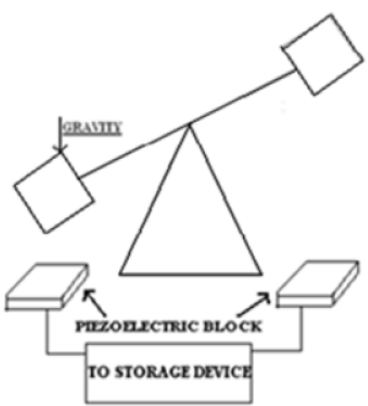

Figure 4: An arrangement using piezoelectric block

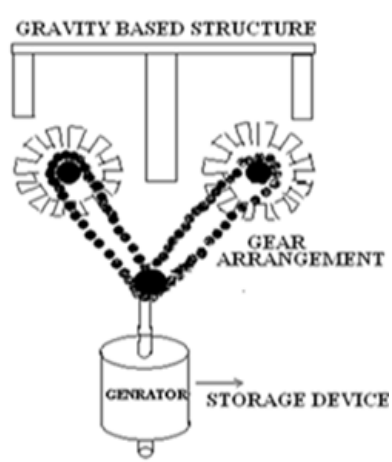

Figure 5: An Arrangement using mechanical structure.

\section{Conclusion}

This concept could be an better option as an source for generation in small captive area. It could be reliable for low voltage needs and can be successfully made possible to enhance the performance of system in future $[5,6]$. The advantages regarding this is that it would not be that much costly, after installation no need to give any input, environment friendly performance, can be used for $24 \times 7$ etc. and have scope in near future.

\section{References}

1. Misner CW, Thorne KS, Wheeler JA (1973) Gravitation, Freeman WH, USA.

2. Alternative Energy News (2016) Alternative energy news, and information about renewable energy technologies.

3. http://challenge.ecomagination.com.

4. Euler (2010) Reprint: Complaints HKSAR misleading election advertising.

5. http://www.firstgravitymachine.com.

6. http://www.eulertruthbible.wordpress.com/.../the-principle-of-generation-ofelect. 